\title{
ON THE ENUMERATION OF SOME D-OPTIMAL DESIGNS
}

\author{
WILLIAM P. ORRICK
}

\begin{abstract}
Two matrices with elements taken from the set $\{-1,1\}$ are Hadamard equivalent if one can be converted into the other by a sequence of permutations of rows and columns, and negations of rows and columns. In this paper we summarize what is known about the number of equivalence classes of matrices having maximal determinant. We establish that there are 7 equivalence classes for matrices of order 21 and that there are at least 9,884 equivalence classes for matrices of order 26 . The latter result is obtained primarily using a switching technique for producing new designs from old.
\end{abstract}

\section{INTRODUCTION}

In this paper we consider exact D-optimal first order saturated designs with $n$ observations, which can be thought of simply as $n \times n$ matrices with entries \pm 1 having maximal determinant. We are concerned with the enumeration and classification of such designs up to equivalence, which is defined below. Our first goal was to compile, from the literature, a list of all known results. The result for $n=11$ seemed to require confirmation, which we undertook as part of our task. Our second goal was to examine orders which have not yet received any attention, $n=21$ and $n=26$ in particular. In order 21 , we find that there are 7 equivalence classes of designs. We have not attempted a complete enumeration in order 26, but we do show that there are at least 9,884 inequivalent designs. The large number of designs is surprising to us considering that there are only 78 designs in order 25 , and 487 in order 28. Many of the new designs in order 26 were obtained from other designs by a method known as switching, the same method that we recently used to obtain the lower bound of 3,578,006 on the number of Hadamard matrices in order 32 [19].

\section{Preliminaries}

Two matrices with elements taken from the set $\{-1,1\}$ are Hadamard equivalent (or simply equivalent) if one can be converted into the other by a sequence of permutations of rows and columns, and negations of rows and columns. If $R$ denotes a \pm 1 matrix of order $n$ and we wish to discover conditions under which $R$ has maximal determinant, it is helpful to consider the Gram matrices $M=R R^{\mathrm{T}}$ and $M^{\prime}=R^{\mathrm{T}} R$.

Date: July 3, 2018.

1991 Mathematics Subject Classification. Primary 05B20, 05B30, 62K05.

Key words and phrases. Maximal determinant, D-optimal design, Hadamard matrix, Hadamard equivalence. 
The notion of equivalence for matrices $R$ carries over to equivalence of the corresponding $M$ and $M^{\prime}$ which are necessarily symmetric. The appropriate notion of equivalence for the latter involves simultaneous permutation of rows and the corresponding columns, and simultaneous negation of rows and the corresponding columns. When $n$ is odd, we may, by normalizing $R$ appropriately, take all entries in $M$ and $M^{\prime}$ to be congruent to $n \bmod$ 4 .

If $M=M^{\prime}=n I$ then $R$ certainly has maximal determinant - it is a Hadamard matrixbut this condition cannot hold unless $n=0,1$, or $n \equiv 0 \bmod 4$.

The largest possible determinant for $n \equiv 1 \bmod 4$ occurs when $R$ is equivalent to a matrix with Gram matrix $M=M^{\prime}=(n-1) I_{n}+J_{n}$ [1]. Such an $R$ is necessarily the \pm 1 incidence matrix of a symmetric balanced incomplete block design with parameters $\left(k^{2}+(k+1)^{2}, k^{2}, k(k-1) / 2\right)$, which implies that $n=k^{2}+(k+1)^{2}$ for some integer $k$. Such designs are known for $n=1,5,13,25$, and many higher orders. For $n=9,17$, and 21 which do not meet the necessary condition for this best possible form, the optimal Gram matrices have been found by computer search. In all three cases, $M=M^{\prime}$ and they differ from the best possible form only in a single row and column, which we may take to be the first. For order 17, the off-diagonal entries in the first row and column all equal -3 rather than 1 [17. For orders 9 and 21, the first row and column contain one 5 and four $5 \mathrm{~s}$ respectively, with the remaining off-diagonal entries equal to 1 [7, 2].

If $n \equiv 2 \bmod 4$ and then the largest possible determinant occurs when $R$ is equivalent to a matrix with Gram matrices $M=M^{\prime}=(n-2) I_{n}+2 I_{2} \otimes J_{n / 2}$ where $J_{k}$ is the $k \times k$ all 1 matrix [6, 26]. Writing

$$
R=\left[\begin{array}{ll}
A & B \\
C & D
\end{array}\right]
$$

with submatrices $A, B, C$, and $D$ of order $n / 2$, we may, by permuting and negating rows and columns if necessary, take the row and column sums of $A$ and $-D$ to be $a$ and the row and column sums of $B$ and $C$ to be $b$ with $a \geq b \geq 0$ and $a^{2}+b^{2}=2 n-2$. Hence a necessary condition on the existence of a design with this best possible $M$ is that $2 n-2$ be expressible as the sum of two squares [6]. For $n \leq 50$ this excludes $n=22$ and 34, but designs do exist for the other orders [6, 27]. The optimal $M$ is not known for $n=22$ or 34.

For $n \equiv 3 \bmod 4$ the best possible $M$ is more complicated [5], and is not known to be achieved in any order. For $n \leq 15$, the optimal $M$ has been found by computer search [8, 18].

\section{KNOWN CLASSIFICATION AND ENUMERATION RESULTS}

In 1946 Williamson [25] found the maximal determinants up to $n=7$ and showed that there is a unique optimal matrix up to equivalence in each order. In his 1988 dissertation [23, Smith states that this can be shown up to $n=10$. Certainly uniqueness of the optimal design for each of these orders, and for $n=12$ and 13, can be established by hand 
without difficulty, especially when the proved optimal forms of $M=M^{\prime}$ are assumed. For other values of $n$, we provide the following survey of known results.

The best studied case is that of Hadamard matrices, and the numbers of inequivalent matrices in orders 16, 20, 24, and 28 are known to be 5, 3, 60, and 487 [9, 10, 12, 13, 14].

In the case $n \equiv 1 \bmod 4$, we have that the number of inequivalent designs in order 25 is 78 4. This result was established by Denniston, who used a switching method (which, as we shall demonstrate, is more generally applicable, in particular to order 26) to generate his list of designs. He established completeness of this list by another method. We will prove that there are 3 designs in order 17 and 7 designs in order 21 up to equivalence. The result in order 17 is a simple consequence of results already in the literature.

The case $n \equiv 2 \bmod 4$ has been studied by Cohn [3] who showed that there is a unique design in order 14 , and that there are 3 distinct designs in order 18 . The next size currently amenable to study is order 26 which we investigate here. We find a lower bound of 9,884 inequivalent matrices. In order 22, for which even the value of the maximal determinant has not been established, 30 distinct matrices have been found by means of a gradient ascent algorithm which all have determinant $195312500 \times 2^{21}$. The latter is the current determinant record, which was discovered by Bruce Solomon and collaborators [22].

The case $n \equiv 3 \bmod 4$ is the most difficult. We discuss $n=11$ below. There is a unique optimal design in order 15 [18. The maximal determinant value is not known with certainty in any higher order. In order 19 the current record, $3411968 \times 2^{18}$, is achieved by matrices which have two distinct forms of $M=M^{\prime}$. One of which was found by Smith [23], the other by Cohn. Orrick and Solomon [21] constructed a third matrix which has the same $M=M^{\prime}$ as Cohn's matrix. No other matrices with determinant equalling the record value are known. In order 23, 14 distinct matrices achieving the current record determinant, $662671875 \times 2^{22}$ [22], have been obtained by gradient ascent. In order 27, a new determinant record, $198087192576 \times 2^{26}$, was recently established by Hiroki Tamura [24]. By switching, 66 equivalence classes have been obtained using Tamura's design as a seed matrix.

Current knowledge is summarized in the table. For those values of $n$ for which the maximal determinant is not known, the current record is listed, with value written in italics.

\section{D-OPtimal Designs OF ORDER 11}

It seems probable that Ehlich proved there are 3 inequvialent matrices of maximal determinant. He certainly showed that there are 3 inequivalent forms that $M=M^{\prime}$ can take. His proof was never published although an account of it appears in a paper of Galil and Kiefer [8]. Nevertheless, there appears to be no clear statement anywhere in the literature that each of these 3 forms decomposes as $M=R R^{\mathrm{T}}$ in an essentially unique way. We have taken the trouble to confirm that this is the case.

The proof could be done by hand, but would be tedious. Instead we adapted some existing programs for the purpose. The backtracking decomposition program used in [18] 
TABLE 1. Number of inequivalent matrices achieving the maximal determinant (current record in italics where maximal determinant is not known).

\begin{tabular}{ll|ll|ll|ll}
$n$ & $N$ & $n$ & $N$ & $n$ & $N$ & $n$ & $N$ \\
\hline 1 & 1 & 2 & 1 & 3 & 1 & 4 & 1 \\
5 & 1 & 6 & 1 & 7 & 1 & 8 & 1 \\
9 & 1 & 10 & 1 & 11 & 3 & 12 & 1 \\
13 & 1 & 14 & 1 & 15 & 1 & 16 & 5 \\
17 & 3 & 18 & 3 & 19 & $\geq 3$ & 20 & 3 \\
21 & 7 & 22 & $\geq 30$ & 23 & $\geq 14$ & 24 & 60 \\
25 & 78 & 26 & $\geq 9884$ & 27 & $\geq 66$ & 28 & 487
\end{tabular}

to study the cases $n=29,33$, and 37 was run on each of Ehlich's 3 matrices $M$. Since our backtracking program does not perform complete isomorph rejection, a set of different matrices $R$ was produced in each case. We then used Brendan McKay's nauty [16, 15] to establish that all matrices in each set are equivalent to each other. Hence there are 3 equivalence classes of maximal determinant matrices in order 11.

\section{D-OPTIMAL DESIGNS OF ORDER 17}

In order 17, it was shown by Moyssiadis and Kounias [17] that the maximal determinant matrix $R$ must be equivalent to a matrix whose Gram matrices $M=M^{\prime}$ have all 16 offdiagonal entries in the first row and in the first column equal to -3 , while all remaining off-diagonal entries equal 1. They noted that, up to overall sign, this implies that the first row and column of $R$ consist entirely of 1 s while the $16 \times 16$ "core" is a regular Hadamard matrix of order 16 . The latter is the \pm 1 incidence matrix of a $2-(16,6,2)$ design. (A regular Hadamard matrix is one with constant row and columns sums, and must have perfect square order.) Husain [11] proved that there are 3 such designs up to isomorphism. From this it follows that there are 3 inequivalent D-optimal designs of order 17.

\section{D-Optimal Designs of ORDer 21}

Chadjipantelis, Kounias, and Moyssiadis found that the optimal matrix $M=M^{\prime}$ is one with four $5 \mathrm{~s}$ in the first row and column, and 1 s everywhere else [2. Orrick and Solomon showed that appending a row and column to a specially normalized $20 \times 20$ Hadamard matrix on which a certain rank-1 update has been performed produces a Doptimal design [20]. This construction is known as the 3-normalized maximal excess construction. The question presents itself: Do there exist optimal matrices that are not derived from this construction?

To answer this, we observe that the condition $R R^{\mathrm{T}}=R^{\mathrm{T}} R=M$ imposes more structure on $R$ than might be supposed. Because of the structure of $M$, we segment $R$ into a $(1+4+16) \times(1+4+16)$ matrix. The row and column sums of the blocks formed by this segmentation become relevant variables. The conditions $M^{2}=R R^{\mathrm{T}} R R^{\mathrm{T}}=R M R^{\mathrm{T}}$ 
and $R M=R R^{\mathrm{T}} R=M R$ imply a set of diophantine equations for these variables, which are also subject to the obvious upper and lower bounds. Solution of sets of equations of this type has been done in several places [17, 18. We omit the proof of the following.

Theorem 6.1. Let $r_{j}$ be the $j^{\text {th }}$ row of $R$ partitioned into $1+4+16$ elements as $r_{j}=$ $\left(a_{j}, b_{j}, c_{j}\right)$ and let $A_{j}, B_{j}$, and $C_{j}$ be the sums of the elements of $a_{j}, b_{j}$, and $c_{j}$. Then $\left(A_{1}, B_{1}, C_{1}\right)=(1,-4,16),\left(A_{j}, B_{j}, C_{j}\right)=(-1,2,8)$ for $2 \leq j \leq 5$, and $\left(A_{j}, B_{j}, C_{j}\right)=$ $(1,2,2)$ for $6 \leq j \leq 21$. The columns of $R$ also have this structure.

By the theorem, we can assume the first 5 rows take the form

$$
\left[\begin{array}{ccccccc}
+ & ---- & ++++ & ++++ & ++++ & ++++ \\
- & -+++ & ---- & ++++ & ++++ & ++++ \\
- & +-++ & ++++ & ---- & ++++ & ++++ \\
- & ++-+ & ++++ & ++++ & ---- & ++++ \\
- & +++- & ++++ & ++++ & ++++ & ----
\end{array}\right]
$$

and the first 5 columns take this form transposed. To determine the remaining $16 \times 16$ submatrix, we partition it into a $4 \times 4$ array of $4 \times 4$ blocks. The inner products of rows 6-21 with rows $2-5$, and the corresponding inner products for columns, force the row and column sums of the $4 \times 4$ blocks to take the values

$$
\left[\begin{array}{llll}
2 & 0 & 0 & 0 \\
0 & 2 & 0 & 0 \\
0 & 0 & 2 & 0 \\
0 & 0 & 0 & 2
\end{array}\right]
$$

Without loss of generality we may now take the form of $R$ to be

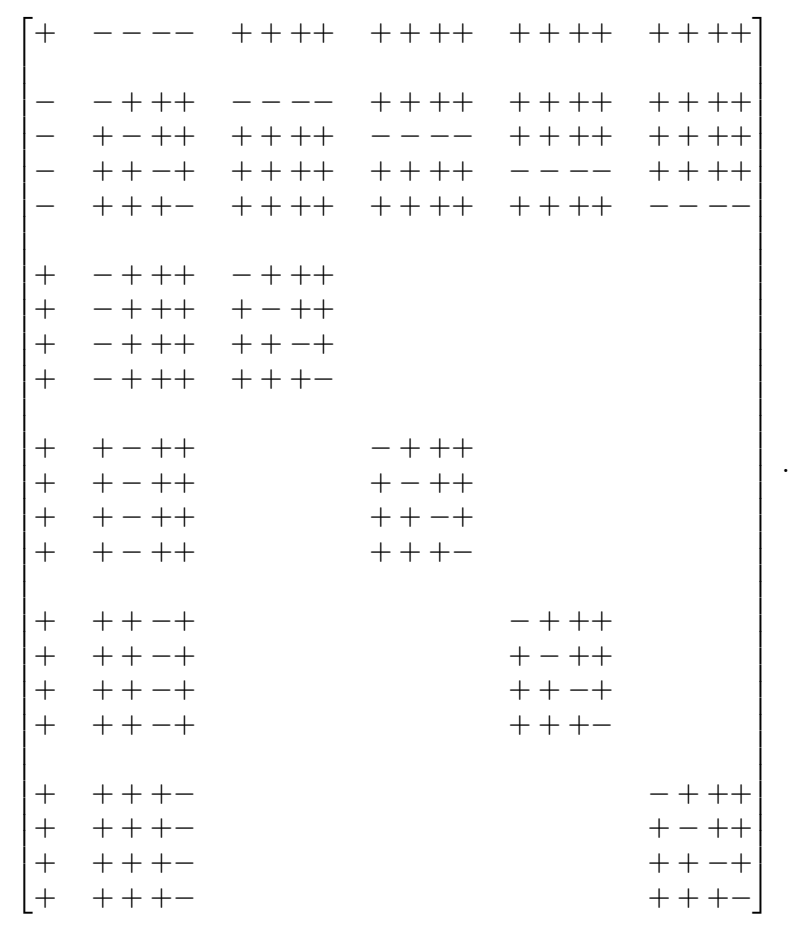


The missing blocks all have row and column sum 0. Before proceeding to find them, we note that if the first column is deleted, then row 1 together with rows 6-21 form an orthogonal set. To complete these 17 rows to a $20 \times 20$ Hadamard matrix, we need only add the 3 rows

$$
\left[\begin{array}{ccccc}
--++ & ---- & ---- & ++++ & ++++ \\
-+-+ & ---- & ++++ & ---- & ++++ \\
-++- & ---- & ++++ & ++++ & ----
\end{array}\right]
$$

The implication is that any D-optimal matrix of order 21 is obtained from a $20 \times 20$ Hadamard matrix by means of the 3-normalized maximal excess construction. This construction amounts to reversing the procedure by replacing the 3 added rows with rows $2-5$ of the above matrix minus their first column, and finally appending column 1 . For details, see [20]. To get a D-optimal matrix, the starting Hadamard matrix must have 3 -normalized excess 76 , which is the largest possible for order 20. It turns out that any 3 -normalized Hadamard matrix of order 20 has row sums $\left(0_{3}, 12,4_{16}\right)$ and hence the maximal excess of 76 . We must therefore consider all possible 3 -normalizations of each of the 3 inequivalent $20 \times 20$ Hadamard matrices [10] in order to determine how many distinct D-optimal designs of order 21 there are. The method we used was the following:

- To each starting Hadamard matrix we applied the 3-normalized maximal excess construction.

- We put the matrix in the form (11) by suitable row and column permutations.

- We further imposed the condition that the $4 \times 4$ block formed by the intersection of rows 6-9 and columns 10-13 (the $(1,2)$ block) and the $4 \times 4$ block formed by the intersection of rows 14-17 and columns 18-21 (the $(3,4)$ block), both of which have row and column sums 0 , be in one of two standard forms:

$$
\left[\begin{array}{l}
--++ \\
-+-+ \\
+-+- \\
++--
\end{array}\right], \quad\left[\begin{array}{l}
--++ \\
--++ \\
++-- \\
++--
\end{array}\right]
$$

- There remain two types of operation that preserve the overall structure we have imposed on the matrix: (1) permutations of the blocks that send diagonal blocks to diagonal blocks, coupled with the permutations of rows $2-5$ and columns $2-$ 5 needed to preserve their form, and (2) automorphisms of the $(1,2)$ and $(3,4)$ blocks, coupled with the transverse permutations needed to maintain the form of the diagonal blocks. We ran through these operations, stopping as soon as the resulting matrix matched one on our canonical list (initially empty). If all possible operations were exhausted without finding a match, the current matrix was added to the canonical list.

By carrying out this procedure, we found 7 distinct forms. These can be found at the website The Hadamard maximal determinant problem [21. 


\section{D-OPTIMAL DESIGNS OF ORDER 26}

There are two classes of D-optimal designs in order 26, relating to the two ways that 50 can be partitioned into two squares, $50=5^{2}+5^{2}$ and $50=7^{2}+1^{2}$. Depending on which of these we take, either all the matrices $A, B, C$, and $-D$ will have row and column sums equal to 5 (the $(5,5)$-type), or $A$ and $-D$ will have row and column sums 7 and $B$ and $C$ will have row and column sums 1 (the $(7,1)$-type).

Two standard constructions produce D-optimal designs in order 26. Construction 16 takes the submatrices $A$ and $B$ to be circulant, and sets $D=-A^{\mathrm{T}}$ and $C=B^{\mathrm{T}}$. The search for the initial rows of $A$ and $B$ can then be done exhaustively. The complete set of solutions, found by Yang [28, includes 3 matrices of $(5,5)$ type one of which is self-dual, the other of which form a dual pair. It also includes 3 matrices of $(7,1)$ type, again consisting of a self-dual matrix and a dual pair.

Construction 2 forms the matrices

$$
R=\left[\begin{array}{cc}
A & B \\
A & -B
\end{array}\right] \quad R^{\prime}=\left[\begin{array}{cc}
A & A \\
B & -B
\end{array}\right]
$$

from maximal determinant matrices $A$ and $B$ of order 13 [26]. $R$ and $R^{\prime}$ are necessarily of $(5,5)$ type. Since there is a unique equivalence class in order $13, A$ and $B$ must be equivalent. By performing certain transformations on $A$ or $B$, however, we can obtain inequivalent matrices $R$. Permuting or negating columns of $A$ or $B$ permutes or negates the corresponding columns of $R$ which does not change its equivalence class. Negating row $j$ of $B$ interchanges rows $j$ and $13+j$ of $R$. Hence negating rows of $A$ or $B$ does not change the equivalence class of $R$. Permuting rows $j$ and $k$ of $B$ may, however change the equivalence class of $R$. We fix $A$, and consider the set of all possible permutations of rows of $B$. By this operation, and by transposition of $R$ we can produce exactly 367 equivalence classes.

Additional matrices have been produced in two ways. One way is to use the gradient ascent method of [22]. Good starting matrices for the ascent are $24 \times 24$ Hadamard matrices, augmented with two random rows and columns, and maximal determinant matrices of order 25, augmented with one random row and column. Matrices of $(5,5)$ type are produced with high probability, whereas $(7,1)$-type matrices are produced only a small fraction of $1 \%$ of the time.

The second way of producing new matrices is called switching and is extremely powerful. Row switching acts on 4 rows of the matrix. These 4 rows must have the property that their Hadamard (element-wise) product is the all 1 vector or its negation. If this is the case, then the columns of the $4 \times 26$ matrix formed by extracting these rows will be of at most 8 different types. Moreover, we consider a column and its negation to be the same type, so there are really only 4 different types. Switching consists of negating all columns of a given type. It preserves D-optimality, but generally produces a matrix inequivalent to the original matrix. Negating any of the 4 different column types produces an equivalent result. This method is equivalent to the method Denniston devised to enumerate $(25,9,3)$ 
designs [4. It was also applied to Hadamard matrices of order $8 k$ in [19] which contains proofs of may of the above statements.

As in [19] we define two matrices to be Q-equivalent if one can be obtained from the other by some sequence of row permutation, row negation, and row switching, combined with the corresponding column operations. The associated equivalence classes are called Q-classes. Since Q-equivalence is weaker than Hadamard equivalence, we can regard the Q-class as composed of Hadamard equivalence classes. A Q-class is self-dual if it is identical to the set of the duals of the matrices contained in it. By means of switching, combined with equivalence checking using nauty [16, 15] we have constructed the Q-classes of all $26 \times 26$ matrices obtained either by Constructions 1 and 2 , or by gradient ascent.

For $(5,5)$-type matrices, the dual pair from Construction 1 and all the matrices from Construction 2 are in the same self-dual Q-class, which has 8,545 elements. That all matrices from Construction 2 lie in the same Q-class can be shown by the same method used to show the analogous property for Hadamard matrices of order 32 [19]. The self-dual matrix from Construction 1 forms a Q-class by itself. There are 8 additional Q-classes which were constructed from matrices found by gradient ascent. Three are singleton Qclasses, one self-dual, two forming a dual pair. Three are of size 4 . One is self-dual and the other two form a dual pair. Two are of size 5 and form a dual pair. These 10 Q-classes together contain 8,571 Hadamard equivalence classes.

For the (7,1)-type matrices, the three matrices from Construction 1 form singleton Q-classes, one of which is self-dual, the other two forming a dual pair. In addition, there is a self-dual Q-class of size 1,310, constructed from matrices found by gradient ascent. Combining these 1,313 matrices with the 8,571 matrices of $(5,5)$-type, we find that there are at least 9,884 inequivalent D-optimal designs of order 26.

Switching is a very general technique. For orders $n \equiv 2 \bmod 4$ we have, in addition to $n=26$, applied it to $n=18$ where we find that all three of Cohn's designs are related by switching. One can show that the row-quadruple structure on which switching acts cannot exist in D-optimal designs of order $n \equiv 6 \bmod 8$, but we expect that whenever $n \equiv 2 \bmod 8$, switching will be applicable. As noted above, switching can also be applied to Hadamard matrices of order $n \equiv 0 \bmod 8$, and was originally applied by Denniston in order 25. We have used it in order 17 to show that all three D-optimal designs are Q-equivalent and in order 19 to show that Cohn's matrix and the matrix found by Orrick and Solomon are Q-equivalent. We also applied it to Tamura's record determinant matrix in order 27 to produce 66 inequivalent matrices.

\section{ACKNowledgments}

I thank Bruce Solomon for many helpful conversations. I used Mathematica extensively during this project and also thank Indiana University for the use of its IBM RS/6000 SP computing platform. 


\section{REFERENCES}

[1] G. Barba. Intorno al teorema di hadamard sui determinanti a valore massimo. Giorn. Mat. Battaglini, 71:70-86, 1933.

[2] Theo Chadjipantelis, Stratis Kounias, and Chronis Moyssiadis. The maximum determinant of $21 \times 21$ $(+1,-1)$-matrices and D-optimal designs. J. Statist. Plann. Inference, 16(2):167-178, 1987.

[3] J. H. E. Cohn. On the number of D-optimal designs. J. Combin. Theory Ser. A, 66(2):214-225, 1994.

[4] R. H. F. Denniston. Enumeration of symmetric designs (25,9,3). In Algebraic and geometric combinatorics, volume 65 of North-Holland Math. Stud., pages 111-127. North-Holland, Amsterdam, 1982.

[5] Hartmut Ehlich. Determinantenabschätzung für binäre Matrizen mit $n \equiv 3 \bmod 4$. Math. Z., 84:438447, 1964.

[6] Hartmut Ehlich. Determinantenabschätzungen für binäre Matrizen. Math. Z., 83:123-132, 1964.

[7] Hartmut Ehlich and Karl Zeller. Binäre matrizen. Z. Angew. Math. Mech., 42:T20-T21, 1962.

[8] Z. Galil and J. Kiefer. D-optimum weighing designs. Ann. Statist., 8(6):1293-1306, 1980.

[9] Marshall Hall, Jr. Research Summary No. 36-10, Volume I, chapter Hadamard matrices of order 16, pages 21-26. Jet Propulsion Laboratory, Pasadena, 1961.

[10] Marshall Hall, Jr. Hadamard matrices of order 20. Technical Report 32-761, Jet Propulsion Laboratory, Pasadena, 1965.

[11] Q. M. Husain. On the totality of the solutions for the symmetrical incomplete block designs: $\lambda=$ $2, k=5$ or 6. Sankhyā, 7:204-208, 1945.

[12] Noboru Ito, Jeffrey S. Leon, and Judith Q. Longyear. Classification of $3-(24,12,5)$ designs and 24-dimensional Hadamard matrices. J. Combin. Theory Ser. A, 31(1):66-93, 1981.

[13] Hiroshi Kimura. New Hadamard matrix of order 24. Graphs Combin., 5(3):235-242, 1989.

[14] Hiroshi Kimura. Classification of Hadamard matrices of order 28. Discrete Math., 133(1-3):171-180, 1994.

[15] Brendan D. McKay. Hadamard equivalence via graph isomorphism. Discrete Math., 27(2):213-214, 1979.

[16] Brendan D. McKay. nauty User's Guide (Version 2.2). Computer Science Department, Australian National University, 2004.

[17] Chronis Moyssiadis and Stratis Kounias. The exact $D$-optimal first order saturated design with 17 observations. J. Statist. Plann. Inference, 7(1):13-27, 1982/83.

[18] William P. Orrick. The maximal $\{-1,1\}$-determinant of order 15. preprint, pages 1-30, 2004. math.CO/0401179, accepted for publication in Metrika.

[19] William P. Orrick. Switching operations for Hadamard matrices. preprint, pages 1-19, 2005. math.CO/0507515.

[20] William P. Orrick and Bruce Solomon. Large-determinant sign matrices of order $4 k+1$. preprint, pages 1-24, 2003. math.CO/0311292.

[21] William P. Orrick and Bruce Solomon. The Hadamard maximal determinant problem. http://www . indiana. edu/ maxdet, 2005.

[22] William P. Orrick, Bruce Solomon, Roland Dowdeswell, and Warren D. Smith. New lower bounds for the maximal determinant problem. preprint, pages 1-15, 2003. math.CO/0304410.

[23] Warren D. Smith. Studies in Computational Geometry Motivated by Mesh Generation. PhD thesis, Princeton University, 1988.

[24] Hiroki Tamura. personal communication, 2005. Hiroki Tamura has informed us of new determinant records in orders 27,31 , and 59 .

[25] John Williamson. Determinants whose elements are 0 and 1. Amer. Math. Monthly, 53:427-434, 1946. 
[26] M. Wojtas. On Hadamard's inequality for the determinants of order non-divisible by 4. Colloq. Math., 12:73-83, 1964.

[27] C. H. Yang. Some designs for maximal $(+1,-1)$-determinant of order $n \equiv 2(\bmod 4)$. Math. Comp., 20:147-148, 1966.

[28] C. H. Yang. On designs of maximal $(+1,-1)$-matrices of order $n \equiv 2(\bmod 4)$. Math. Comp., 22:174$180,1968$.

Department of Mathematics, Indiana University, Bloomington IN 47405, USA 